\title{
Cryogenic Temperature Response of Reflection-Based Phase-Shifted Long-Period Fiber Gratings
}

\author{
Raquel Martins, Paulo Caldas, Bruno Teixeira, João Azevedo, José Monteiro, João H. Belo, João P. Araújo, \\ José L. Santos, and Gaspar Rego
}

\begin{abstract}
In this study, we investigated the temperature behavior of phase-shifted long-period fiber gratings (PS-LPFGs) inscribed in two types of optical fiber: B/Ge and SMF28. The experiments were carried out from 5 to $305 \mathrm{~K}$ using a superconducting quantum interference device magnetometer. The average temperature sensitivity obtained of $-0.43 \mathrm{~nm} / \mathrm{K}$ for PS-LPFGs inscribed in the $\mathrm{B} / \mathrm{Ge}$ fiber is one order of magnitude larger than for PS-LPFGs inscribed in the SMF28 fiber, in the 60-240 K range. Values ranging from $-0.08 \mathrm{~nm} / \mathrm{K}$ up to $0.2 \mathrm{~nm} / \mathrm{K}$ were obtained in the 5-35 K temperature range, which are considerably better than previous results achieved for metal-coated FBGs and also for LPFGs inscribed in a similar B/Ge codoped fiber. Nevertheless, further work is required in order to correctly address sensor reliability.
\end{abstract}

Index Terms-Cryogenic temperatures, long-period fiber grating, optical fiber sensor.

\section{INTRODUCTION}

$\mathbf{S}$ YSTEMS operating at cryogenic temperatures are becoming increasingly important in the energy sector, transportation, and medicine technology. Cryogenic fuels such as liquid hydrogen (also employed in aerospace vehicles), oxygen and liquefied natural gas are often considered as major energy alternatives to fossil fuels. To ensure the safe storage, transfer and dispensing of liquefied fuels, highly sensitive and reliable

Manuscript received September 14, 2014; revised November 3, 2014 and December 8, 2014; accepted December 9, 2014. Date of publication December 17, 2014; date of current version April 29, 2015.

R. Martins is with Institute for Systems and Computer Engineering of Porto Technology and Science, 4169-007 Porto, Portugal, with the Instituto Politécnico de Viana do Castelo, ESTG, 4900-3487 Viana do Castelo, Portugal, and also with the Departamento de Física e Astronomia da, Faculdade de Ciencias da Universidade do Porto, 4099-002 Porto, Portugal (e-mail: rmartins@estg.ipvc.pt).

P. Caldas, B. Teixeira, J. Azevedo, and G. Rego are with the Institute for Systems and Computer Engineering of Porto Technology and Science, 4169007 Porto, Portugal, and also with the Instituto Politécnico de Viana do Castelo, ESTG, 4900-347 Viana do Castelo, Portugal (e-mail: pcaldas@estg.ipvc.pt; brunotx1@gmail.com; azev_qwerty@hotmail.com; gmrego@fc.up.pt).

J. Monteiro is with FiberSensing, 4470-640 Maia, Portugal (e-mail: zemmonteiro@gmail.com).

J. H. Belo and J. P. Araújo are with Instituto de Física dos Materiais da Universidade do Porto, IN-Institute of Nanoscience and Nanotechnology, 4169007 Porto, Portugal, and also with the Departamento de Física e Astronomia da Faculdade de Ciencias da Universidade do Porto, 4099-002 Porto, Portugal (email: joao.horta.belo@gmail.com; jearaujo@fc.up.pt).

J. L. Santos is with Institute for Systems and Computer Engineering of Porto Technology and Science, 4169-007 Porto, Portugal, and also also with Departamento de Física e Astronomia da Faculdade de Ciencias da Universidade do Porto, 4099-002 Porto, Portugal (e-mail: josantos@fc.up.pt).

Color versions of one or more of the figures in this paper are available online at http://ieeexplore.ieee.org.

Digital Object Identifier 10.1109/JLT.2014.2381236 sensor networks are required to provide continuous monitoring of multiple parameters at multiple locations for on-demand, real-time fuel management in cryogenic environments [1], [2]. On the other hand, cryogenic systems based on superconducting magnets find application, among other fields, in medicine for magnetic resonance imaging and also in nuclear fusion energy production, as in the case of the ITER Project [3]. In this context, the hot-spot temperature, the thermal stress and the maximum pressure in the cable in conduit conductors and the risk of helium explosion are some parameters to be measured [4]-[6]. Furthermore, these cryogenic systems with high intensity magnetic fields demand for monitoring devices shielded to the presence of those fields and, simultaneously, that are able to prevent heat transfer from their lead wires. Therefore, optical fiber sensors being dielectric and low size, immune to electromagnetic interference and having low thermal conductivity are good candidates to fulfil these requirements allowing the measurement of important parameters, such as, temperature and strain in all critical system components.

Optical fiber sensors and, namely, those based on fiber Bragg gratings (FBG), due to their intrinsic properties, have already proved their excellence in structural health monitoring at room temperatures [7]. FBGs have a sub-micron period and act to couple light from the fundamental forward-propagating mode of the optical fiber to a backward, counter-propagating mode. This coupling occurs at a specific wavelength that satisfies the Bragg resonance condition and depends on the grating period and other parameters like temperature and strain. However, as far as temperature measurements are concerned FBGs are essentially insensitive below $100 \mathrm{~K}$. Therefore, several techniques have been applied in order to enhance their thermal sensitivity, such as, by deposition of metal or sol-gel coatings on the fiber cladding or by embedding or bonding them to substrates with very different thermal expansion coefficients [8], [2]. When compared to conventional FBGs, the fabrication of the modified ones is time consuming and the temperature sensitivity improvement is limited.

A long-period fiber grating (LPFG) is a periodic structure, with periods typically in the range of $100 \mu \mathrm{m}$ to $1 \mathrm{~mm}$, inscribed in a fiber, which couples light between the core mode and copropagating cladding modes at specific resonance wavelengths. The grating transmission spectrum contains a series of attenuation bands centered at these discrete resonance wavelengths, each attenuation band corresponding to coupling of a different cladding mode. The grating behaves as a selective filter, 
where the resonance wavelengths depend on the period of the LPFG and also on physical parameters, such as temperature, strain, external refractive index and bending radius. LPFGs can, therefore, be used as sensors of these parameters. The thermal behavior of LPFGs from room temperature up to $1500 \mathrm{~K}$ is well documented and it is known that their temperature sensitivity is an order of magnitude higher than for FBGs [9], [10]. On the other hand, results on the thermal behavior of LPFGs at cryogenic temperatures are scarce, despite the very promising results obtained in 2003 by James et al. [11]. In their work, a LPFG inscribed in a $\mathrm{B} / \mathrm{Ge}$ co-doped fiber was able to discriminate temperature differences down to $20 \mathrm{~K}$ and presented a temperature sensitivity of the order of $-0.2 \mathrm{~nm} / \mathrm{K}$ for temperatures above $77 \mathrm{~K}$. These results are better than the ones obtained by FBGs even for modified FBGs (coated or embedded/bonded in structures). One possible explanation for this minute interest on LPFGs-based cryogenic temperature sensors is related to interrogation issues since these gratings work in transmission and, therefore, require access to both ends of the fiber which may be a drawback for some applications. In 2011 [12], we mitigate this problem by proposing a sensor that comprised a LPFG inscribed in the SMF28 fiber spliced to a short piece of Bendbright fiber, from Draka, that allowed small curvature radius. This way, we have implemented a sensor to monitor cryogenic temperatures by inserting the fiber containing the grating in a glass tube with an internal diameter of $4 \mathrm{~mm}$. The temperature sensitivity obtained for temperatures above $77 \mathrm{~K}$ was similar to the values obtained at room temperature, i.e., $\sim 60 \mathrm{pm} / \mathrm{K}$.

Recently, Huang et al. [13] proposed the fabrication of a phase-shifted LPFG (PS-LPFG) by deposition of a silver-mirror at the end-face of the fiber at a distance of the grating-end corresponding to a quarter of the period. This configuration enabled the LPFG to work in reflection. Afterwards, we have implemented a compact sensor head based on a PS-LPFG arcinduced in a standard fiber [14]. The thermal behavior of this sensor, working in reflection, was investigated from $77 \mathrm{~K}$ up to room temperature and results show that its temperature sensitivity is higher than the ones obtained for FBGs, even for those especially prepared to have improved sensitivity.

In this work we have investigated the temperature behavior of PS-LPFGs inscribed in two types of optical fiber: B/Ge and SMF28. The experiments were carried out from 5 to $305 \mathrm{~K}$ using a SQUID magnetometer. In the 60-240 $\mathrm{K}$ temperature range, an average temperature sensitivity of $-0.43 \mathrm{~nm} / \mathrm{K}$ was obtained for PS-LPFGs inscribed in the B/Ge fiber which is one order of magnitude larger than for PS-LPFGs inscribed in the SMF28 fiber. In the $5-35 \mathrm{~K}$ range, we were also able to discriminate temperature changes and, in which concerns the temperature sensitivity, results are considerably better than previous ones achieved for metal-coated FBGs and also for LPFGs inscribed in a similar B/Ge co-doped fiber [8], [11].

\section{THEORY}

For an LPFG, the wavelength at which the mode coupling occurs is given by:

$$
\lambda_{\text {res }}=\left(n_{c o}^{\mathrm{eff}}-n_{c l, m}^{\mathrm{eff}}\right) \Lambda
$$

where $\lambda_{\text {res }}$ represents the resonance wavelengths, $\Lambda$ is the grating period and $n_{c o}^{\mathrm{eff}}$ and $n_{c l, m}^{\mathrm{eff}}$ are the effective refractive indices of the core mode and the $m$ th cladding mode, respectively. For a given fiber, the grating period determines the cladding modes to which light can be coupled [9].

A PS-LPFG arises from changes in the optical phase at specific locations of the grating, in particular, due to modifications of the grating period or of the refractive index. Their theoretical analysis based on the F-matrix method and on analytical expressions were first presented, respectively, by Ke et al. [15] and by Liu et al. [16]. PS-LPFGs can be produced by changing the grating period at specific locations, for instance by creating a gap $L_{\mathrm{p}}$ between two grating periods would result in a phase-shift $\Delta \Phi=2 \pi L_{\mathrm{p}} / \Lambda$. A $L_{\mathrm{p}}=\Lambda / 2$ in the middle of the grating would result in a $\pi$-shifted LPFG which is characterized by a bandpass filter (one peak in-between two dips) centered at each resonance wavelength of the standard LPFG [17]. As mentioned above, if the fiber is cleaved at a distance of $\Lambda / 4$ from the grating, Fresnel reflection would give rise to a $\pi$-shifted LPFG working in reflection. PS-LPFGs can also be produced by exposing a grating section to UV-radiation or to arc-discharges, these would induce permanent localized refractive index changes [10]. Similarly, temporary PS-LPFGs can be attained by localized heating through a resistive heater [18], [19].

The temperature sensitivity of an LPFG is expressed by the following equations [20]:

$$
\frac{d \lambda_{\text {res }}}{d T}=\lambda_{\text {res }} \gamma\left(\alpha+\Gamma_{\text {Temp }}\right)
$$

where $\alpha$ is the thermal expansion coefficient of the fiber. $\gamma$, the general sensitivity factor, describes the waveguide dispersion and is expressed by

$$
\gamma=\frac{\frac{d \lambda_{\mathrm{res}}}{d \Lambda}}{n_{c o}^{\mathrm{eff}}-n_{c l, m}^{\mathrm{eff}}}
$$

where $\mathrm{d} \lambda_{\text {res }} / \mathrm{d} \Lambda$ represents the slope of the dispersion curves. $\Gamma_{\text {Temp }}$ describes the temperature dependence of the waveguide dispersion and is expressed by

$$
\Gamma_{\mathrm{Temp}}=\frac{\xi_{c o} n_{c o}^{\mathrm{eff}}-\xi_{c l} n_{c l, m}^{\mathrm{eff}}}{n_{c o}^{\mathrm{eff}}-n_{c l, m}^{\mathrm{eff}}}
$$

where $\xi_{c o}$ and $\xi_{c l}$ are the thermo-optic coefficients of the core and cladding materials.

The analysis of the four equations enables one to conclude that far from the turning points, for instance by considering grating periods above $300 \mu \mathrm{m}$, the temperature sensitivity increases as the grating period increases (from (1)). $\gamma$ is positive since $n_{c o}^{\text {eff }}$ is greater than $n_{c l, m}^{\mathrm{eff}}$, and $\mathrm{d} \lambda_{\text {res }} / \mathrm{d} \Lambda$ is also positive and increases as the grating period decreases (it depends on the order of the cladding mode). At temperatures above $\sim 120 \mathrm{~K}, \alpha$ is positive and, therefore, the resonance wavelengths will shift towards longer wavelengths with increasing temperature if $\Gamma_{\text {Temp }}$ is at least greater than the symmetric of $\alpha$ (from (2)). For standard Ge-doped silica fibers, as the SMF28 fiber, $\xi_{c o}$ is greater than $\xi_{c l}$ and therefore $\mathrm{d} \lambda_{\text {res }} / \mathrm{d} T$ is also positive. For B/Ge co-doped fibers the sign of $\mathrm{d} \lambda_{\text {res }} / \mathrm{d} T$ will depend on the concentration of $\mathrm{B}_{2} \mathrm{O}_{3}$ in the core since the thermo-optic coefficient decreases as 


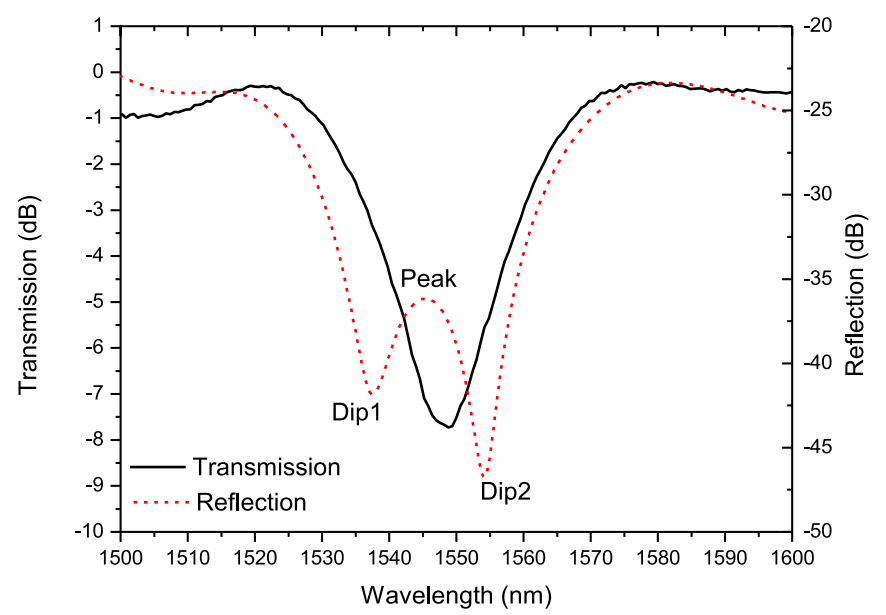

Fig. 1. LPFG transmission spectrum and PS-LPFG reflection spectrum for the grating inscribed in the SMF28 fiber.

the concentration increases. For the Fibercore fiber used in this work $\mathrm{d} \lambda_{\text {res }} / \mathrm{d} T$ is negative from room temperature up to about $650 \mathrm{~K}[10]$.

Thus, by an appropriate choice of the LPFG period it is possible to balance the contributions of the temperature sensitivity to produce a temperature-independent resonance band and also to produce attenuation bands with temperature sensitivities (positive or negative) appropriate to specific applications. Altering the fiber composition, such that the thermo-optic coefficient of the core is either larger or smaller than that of the cladding, can also be used to obtain the desired temperature sensitivity [21].

In which concerns the thermal behavior of PS-LPFGs, it should be mentioned that the resonance wavelengths of the two dips and peak depend on the phase-shift which in turn depends on temperature. A comparison between the temperature sensitivity of a LPFG and of a PS-LPFGs written in the SMF28 fiber, from room temperature up to $500 \mathrm{~K}$, shows an increase of about $10 \%$ for the latter [10], [22]. This can be a consequence of the dependence of the phase-shift on temperature through the thermo-optic coefficient. A considerable change on the phaseshift can be observed in Fig. 6(b) for the PS-LPFG written in the $\mathrm{B} / \mathrm{Ge}$ fiber between $5 \mathrm{~K}$ and room temperature.

\section{EXPERIMENTAL SETUP}

The reflection-based PS-LPFGs were fabricated in-house using the electric-arc technique [10]. First, several gratings with resonances in the third telecommunication window were fabricated with different attenuation. From a series of experiments we concluded that an attenuation of $\sim 7 \mathrm{~dB}$ leads to a better spectrum which is in accordance to [17]. Therefore, we have chosen a LPFG inscribed in the SMF28 fiber with a grating period of $540 \mu \mathrm{m}$ (fabrication parameters: electric current of $9 \mathrm{~mA} ; 1 \mathrm{~s}$ arc duration; $5 \mathrm{~g}$ pulling weight; 44 arc discharges) in order to produce a resonance wavelength at approximately $1550 \mathrm{~nm}$, with $-7 \mathrm{~dB}$ of attenuation (see Fig. 1) and a LPFG inscribed in the $\mathrm{B} / \mathrm{Ge}$ co-doped fiber with a grating period of $540 \mu \mathrm{m}$ (fabrication parameters: electric current of $9 \mathrm{~mA} ; 0.5 \mathrm{~s}$

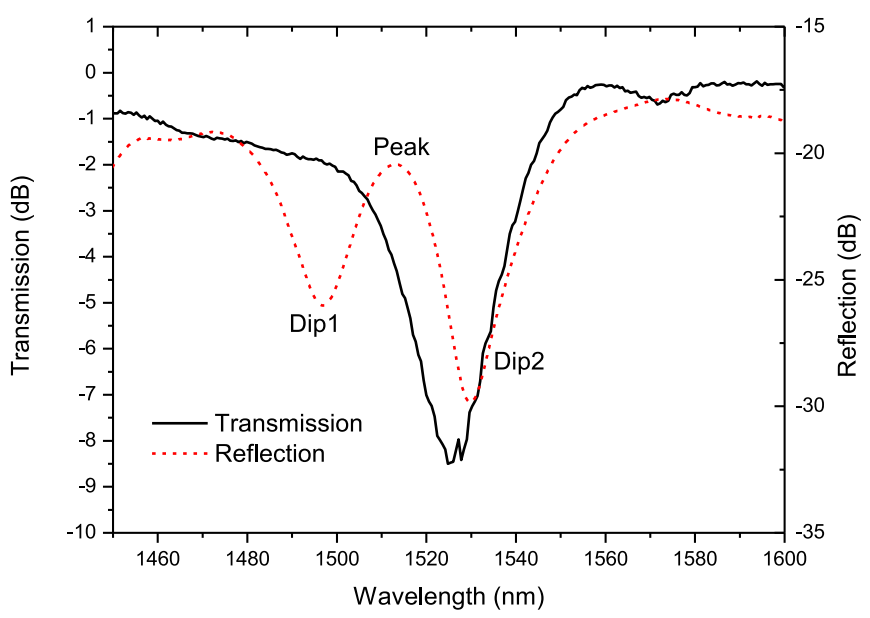

Fig. 2. LPFG transmission spectrum and PS-LPFG reflection spectrum for the grating inscribed in the $\mathrm{B} / \mathrm{Ge}$ co-doped fiber.

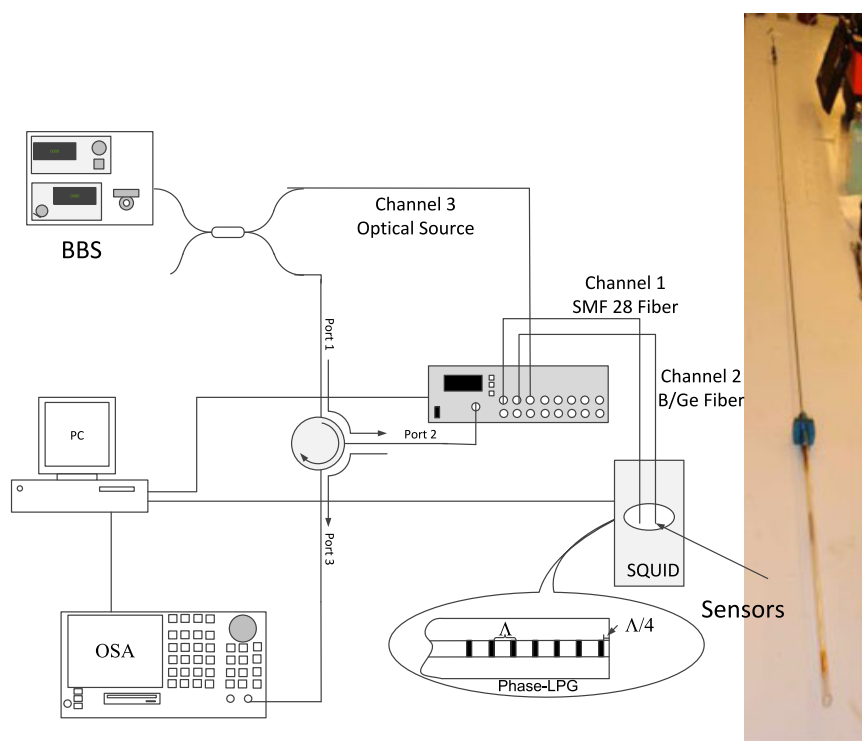

Fig. 3. Scheme of the experimental set-up.

arc duration; $23 \mathrm{~g}$ pulling weight; 26 arc discharges) in order to produce a resonance wavelength at approximately $1525 \mathrm{~nm}$, with $-7 \mathrm{~dB}$ of attenuation (see Fig. 2). Afterwards, the fibers were cleaved near the grating and placed inside a capillary to control the polishing process. The end-face was polished until two resonant dips appeared in the reflection spectrum and had almost equal attenuation strength (see Figs. 1 and 2).

The experimental set-up used to study the thermal behavior of the reflection-based PS- LPFGs is represented in Fig. 3. A Fiber Coupled SLD source (Thorlabs), centered at $1525 \mathrm{~nm}$, was used to illuminate the PS-LPFGs and their spectra were monitored with an optical spectrum analyzer (OSA: Yokogawa AQ6370C). A circulator was used to make the connection between the optical source, the PS-LPFGs and the OSA. It was also used an optical switch that allowed to record the spectrum of the PS-LPFGs and of the optical source. A 50/50 coupler placed between the source, the optical switch and the circulator, 
was used in order to record the spectrum of the optical source, allowing the real time normalization of the PS-LPFGs spectra.

The PS-LPFGs were inserted in a polyimide capillary tube (inside diameter: $1 \mathrm{~mm}$; thermal conductivity: $0.15 \mathrm{~W} /(\mathrm{m} \cdot \mathrm{K})$ ), sealed at both ends (to avoid contact with the helium vapor), and then into a special feed-through apparatus (designed by FiberSensing), which was afterwards inserted into the sample camera of the SQUID (from Quantum Design). The temperature control is obtained through the cooling annulus that enwraps the sample space and where a flux of helium vapor circulates allowing for a homogeneous temperature distribution inside the sample space, monitored by two thermocouples separated by $5 \mathrm{~cm}$. The heating is supplied by an electric circuit surrounding the camera, through the joule effect.

The several optical devices were synchronized with the SQUID magnetometer and their performance was computer controlled by implementing a software program.

\section{RESULTS AND DisCUSSION}

The spectra of the reflection-based PS-LPFGs were recorded between 5 and $305 \mathrm{~K}$, in steps of $10 \mathrm{~K}$. The temperature changed at a rate of $10 \mathrm{~K} / \mathrm{min}$, plus $10 \mathrm{~min}$ wait for temperature to stabilize in the SQUID followed by $1 \mathrm{~h}$ wait for the fiber temperature stabilize. Fig. 4 shows the spectra of the PS-LPFGs inscribed in both fibers at different temperatures. It should be noticed that during the experiment we detected that due to an electric problem, the optical switch did not changed correctly the channels and, therefore, we had to turn back twice to lower temperatures. We assumed that some observed instability, for instance the spectrum at $25 \mathrm{~K}$ in Fig. 4(a) and at $65 \mathrm{~K}$ in Fig. 4(b), was due to that fact. This is more evident in Fig. 7(b) (to be discussed below) where clearly $1 \mathrm{~h}$ was not enough to reach thermal equilibrium at $115 \mathrm{~K}$ after turning back, and, therefore, we repeated the experiment eight weeks later. This time the temperature was increased from 5 to $50 \mathrm{~K}$ in steps of $5 \mathrm{~K}$, up to $160 \mathrm{~K}$ in steps of $10 \mathrm{~K}$, then up to $220 \mathrm{~K}$ in steps of $20 \mathrm{~K}$ and afterwards in steps of $10 \mathrm{~K}$ up to $300 \mathrm{~K}$. It should be noticed that the experiment was done in a semi-automatic way and that when we measured the spectrum at $35 \mathrm{~K}$, after a standby overnight of $7 \mathrm{~h}$, we had only one resonance, the double resonance only recovered at $70 \mathrm{~K}$. Fig. 5 shows the spectra of the PS-LPFG at $50 \mathrm{~K}$ during the second and third set of experiments. During the second experiment only one resonance appeared and its wavelength has no relation with the resonance wavelengths of the two Dips and Peak obtained outside the temperature range 35-60 K (not presented in Fig. 7). Therefore, after reaching the $300 \mathrm{~K}$, the system was cooled down to $5 \mathrm{~K}$ overnight, and we have repeated the experiment again between 5 and $60 \mathrm{~K}$. During this third set we retrace the resonance positions of the previous experiment, for temperatures in the 5 to $30 \mathrm{~K}$ range and we recovered the PS-LPFG spectra between the 35-60 K temperature range (the resonance wavelengths of the two Dips and Peak are shown in Fig. 7). As can be observed in Fig. 5 we recovered the PS-LPFG spectra at $50 \mathrm{~K}$, although in the case of the B/Ge doped fiber, due to limitations of the optical power source, a smooth fitting

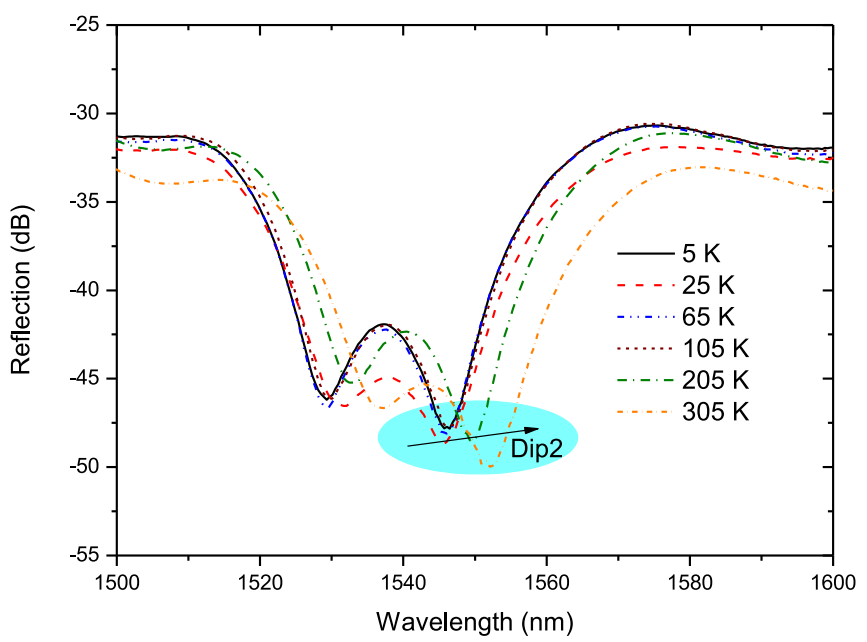

(a)

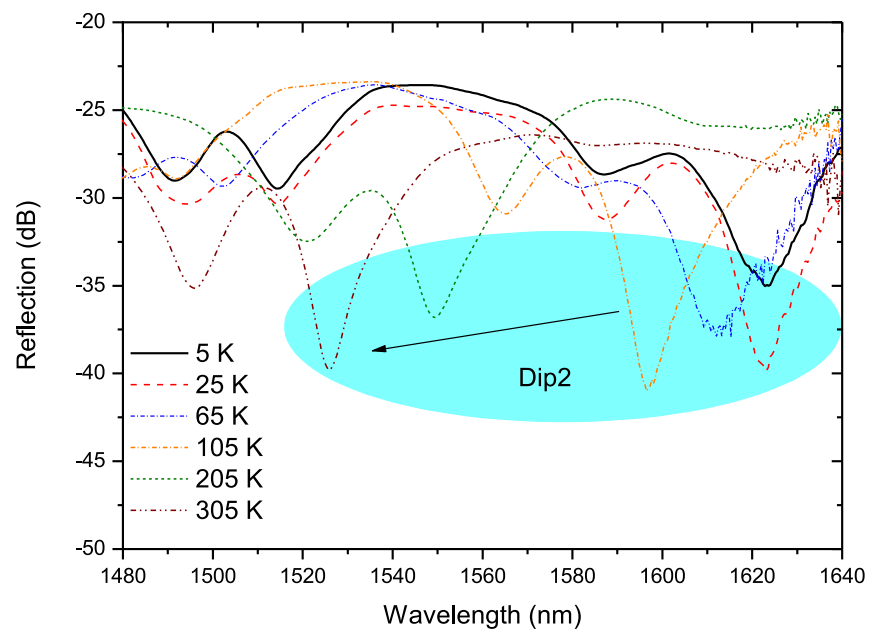

(b)

Fig. 4. Reflection spectra of the PS-LPFG, inscribed in the (a) SMF28 fiber (b) $\mathrm{B} / \mathrm{Ge}$ co-doped fiber, at several temperatures.

was applied to the spectrum in order to detect the resonance wavelength of Dip2.

Fig. 6 shows the spectra of the PS-LPFGs at different temperatures during the second set of experiments. In comparison with Fig. 4, it can be observed that the spectrum of PS-LPFG fabricated in the SMF28 fiber changed considerably in particular in which concerns the reduction in amplitude of the first resonance. In this context the PS-LPFG fabricated in the $\mathrm{B} / \mathrm{Ge}$ co-doped fiber seems to be more stable and the only remark is to the noise level in the long wavelength range which causes an increasing difficulty to the measurement of the resonance wavelength, belonging to the second dip, at lower temperatures. It should be stressed that since we are dealing with phase-shifted gratings it is expected that the temperature affects their spectrum, which means that the change in the resonance positions is a consequence not only of the changes in the physical parameters discussed in Section II but also a result of changes in the optical phase [16].

Fig. 7 shows the temperature dependence of the resonance wavelengths for the PS-LPFGs written in both fibers. As can 


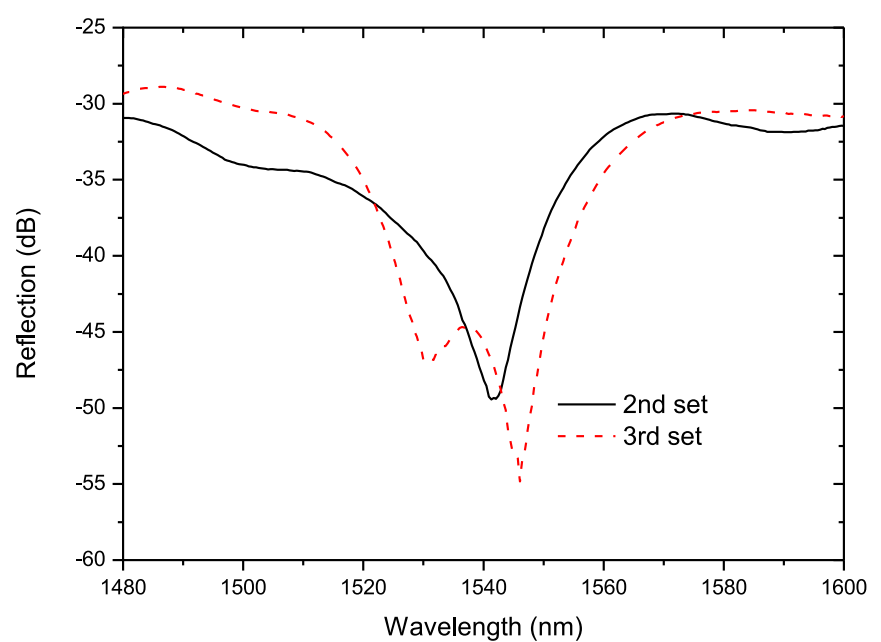

(a)

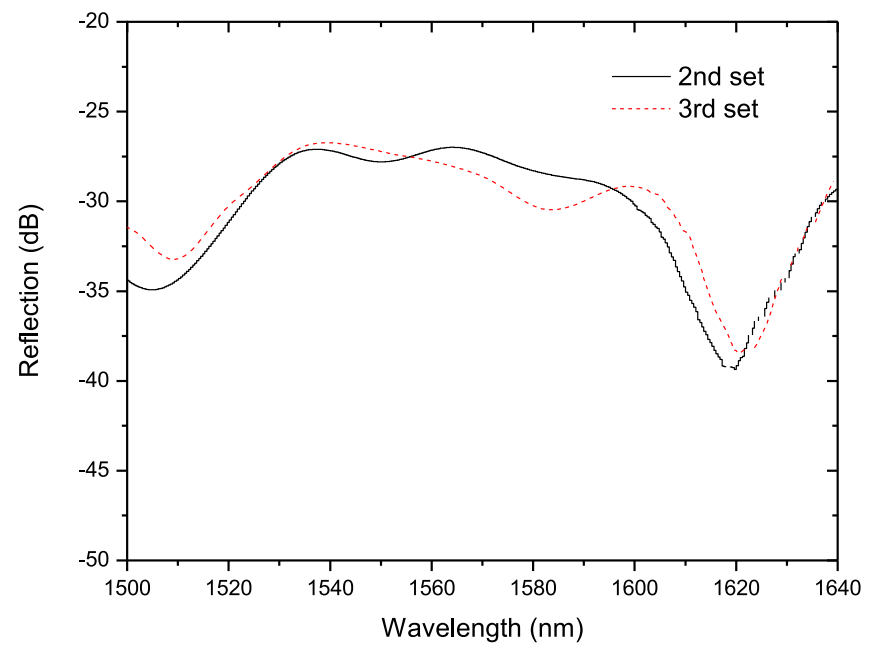

(b)

Fig. 5. Reflection spectra of the PS-LPFG, inscribed in the (a) SMF28 fiber (b) $\mathrm{B} / \mathrm{Ge}$ co-doped fiber, at $50 \mathrm{~K}$ (second and third set of experiments).

be seen in Fig. 7(a) for the SMF28 fiber, except for the first resonance, the curves belonging to the different experiments correlates fairly well. The deviation obtained for the first resonance may be a consequence of the observed change in the first dip in-between the two set of experiments. The good correlation is also true for the PS-LPFG inscribed in the B/Ge co-doped fiber except up to $30 \mathrm{~K}$ (see Fig. 7(b)). Based on the thermal behavior followed by the resonance curves, one may conclude that, in this temperature range, the fiber did not reach thermal equilibrium despite being for several hours at such low temperatures. On one hand, we monitored the spectrum of the PS-LPFGs at $10 \mathrm{~K}$ during the stabilization procedure and we observed that the spectrum was essentially the same after the stabilization of the SQUID temperature. On the other hand, it was observed that during the change from 80 to $90 \mathrm{~K}$ it took the system about $20 \mathrm{~min}$ to stabilize when usually it takes $1 \mathrm{~min}$. Thus in Fig. 7(b), for $90 \mathrm{~K}$ it can be seen a difference of about $10 \mathrm{~nm}$ between the two data points leading to the conclusion that in this case the fiber was at a higher temperature. Therefore, further work is required in order to explain some unpredictable modifications

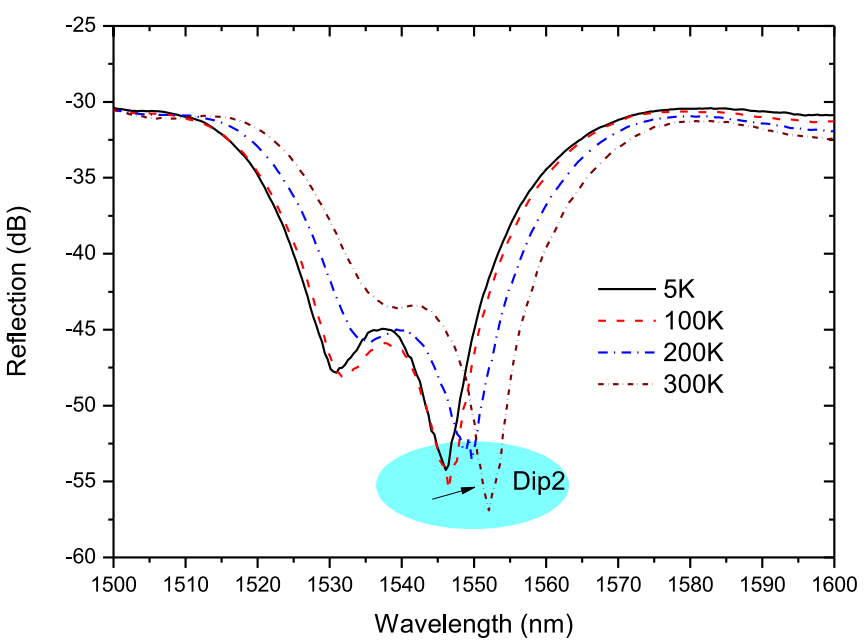

(a)

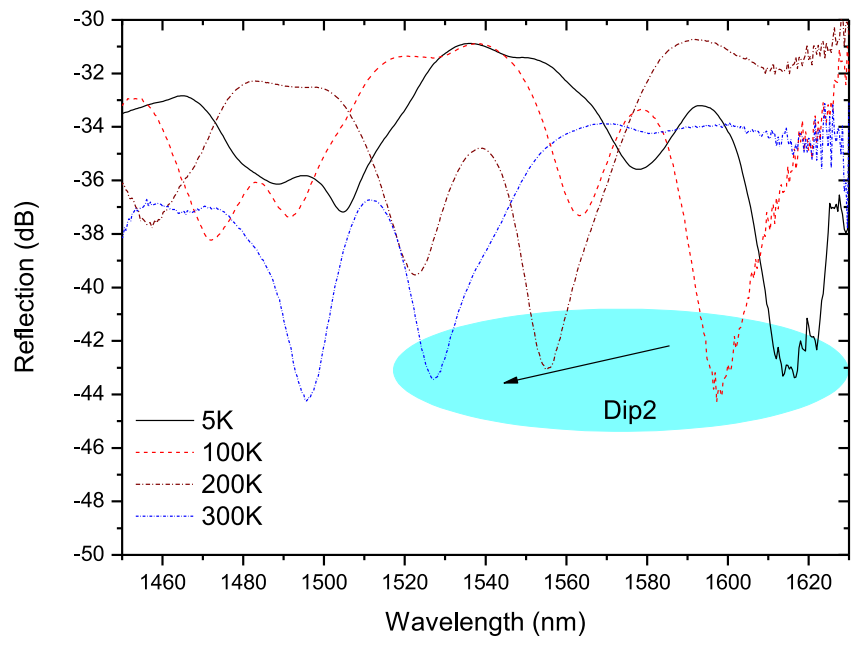

(b)

Fig. 6. Reflection spectra of the PS-LPFG, inscribed in the (a) SMF28 fiber (b) B/Ge co-doped fiber, at several temperatures.

in the spectra that occurred during these experiments. Note, however, that some spectra were taken in the limits of the optical power source leading to optical noise which prevents good measurements. Moreover, we observed some optical instability that depends on the wavelength, which may be related to the fact that the source is partially polarized. Therefore, this fact may also contribute to measurement errors since some optical devices including the PS-LPFGs are polarization sensitive.

In Fig. 7(a), up to a temperature of about $50 \mathrm{~K}$ the resonance wavelength is essentially insensitive, afterwards as the temperature increases the resonance shifts towards longer wavelengths. This behavior can be explained taking into account (1) to (4). The temperature sensitivity depends on the difference between the thermo-optic coefficients of the core and cladding which for the SMF28 fiber is of the order of $2-3 \times 10^{-7} \mathrm{~K}^{-1}$ (assuming a $3.3 \mathrm{~mol} \%$ of $\mathrm{GeO}_{2}$ dopant in the core [23] and considering values for $\mathrm{d} n / \mathrm{d} T$ of $1.94 \times 10^{-5}$ and $1.02 \times 10^{-5} \mathrm{~K}^{-1}$ for pure $\mathrm{GeO}_{2}$ and $\mathrm{SiO}_{2}$, respectively [24]) and on the thermal expansion coefficient of the fiber which takes a common value of $5.5 \times 10^{-7}$ at room temperature. However, calculations show 


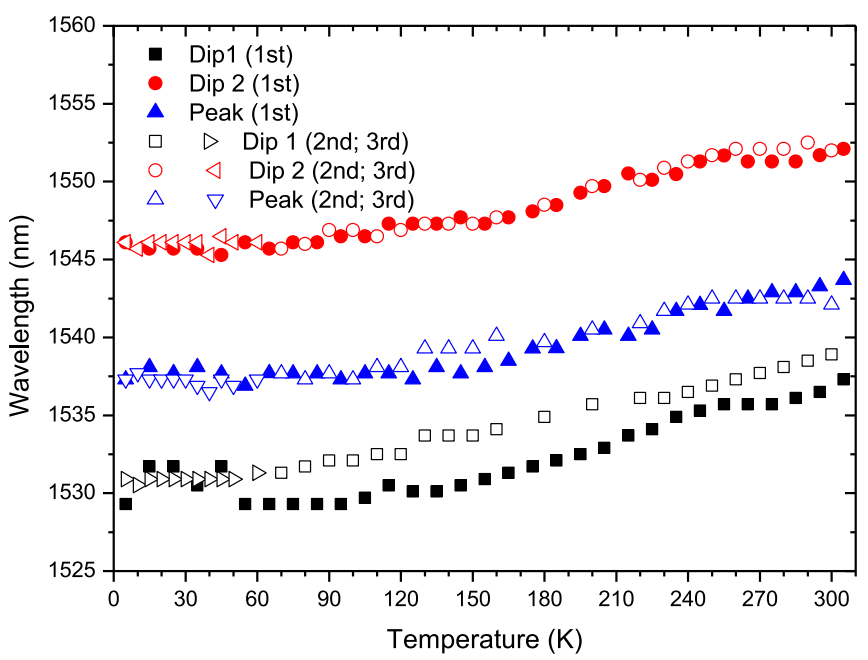

(a)

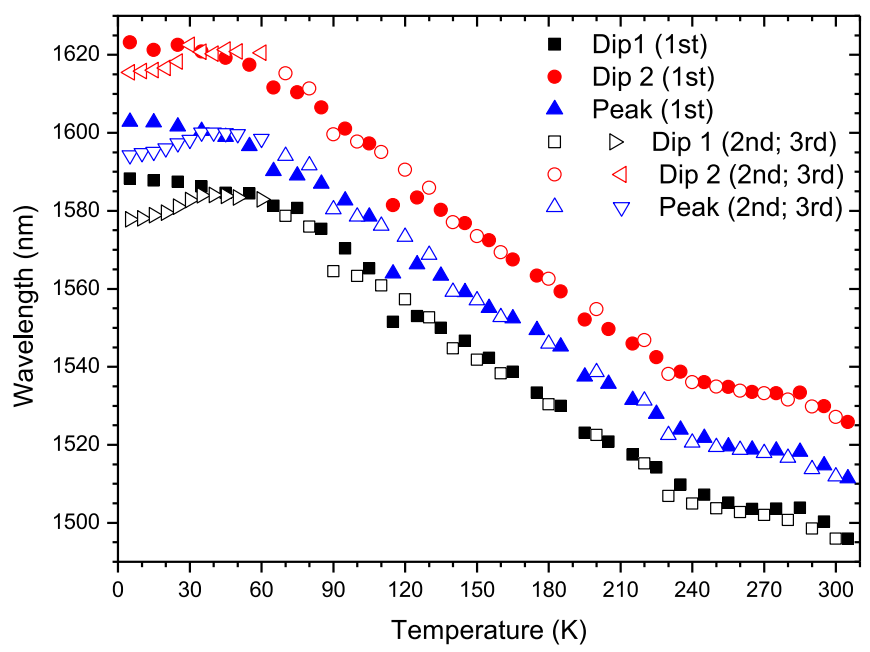

(b)

Fig. 7. Wavelength of the two Dips and the Peak of the PS-LPFG, inscribed in the (a) SMF28 fiber (b) B/Ge co-doped fiber, as a function of temperature.

that the thermal expansion coefficient contributes about $1 \%$ for the temperature sensitivity and, therefore, it can be ignored. The temperature behavior of both parameters can be found in [25], [26]. At cryogenic temperatures the thermo-optic coefficients are about one order of magnitude lower which impacts accordingly the grating temperature sensitivity. The thermo-optic coefficients increase with the temperature increase but at a lower rate above $\sim 200 \mathrm{~K}$. The average temperature sensitivity obtained is $30 \mathrm{pm} / \mathrm{K}$, for the $50-300 \mathrm{~K}$ range.

As can be observed in Fig. 7(b), after $60 \mathrm{~K}$, as the temperature increases the resonance wavelength moves towards shorter wavelengths. This a consequence of the fact that glasses doped with $\mathrm{B}_{2} \mathrm{O}_{3}$ have larger thermal expansion coefficients and lower thermo-optic coefficients [27]-[30]. Therefore, the core thermooptic coefficient of the $\mathrm{B} / \mathrm{Ge}$ co-doped fiber is substantially lower than that of the silica cladding $\left(\mathrm{d} n / \mathrm{d} T\right.$ is $-3.50 \times 10^{-5} \mathrm{~K}^{-1}$ for pure $\mathrm{B}_{2} \mathrm{O}_{3}$ [24]). For the fiber composition presented in [23], we estimated a value which is about $20 \%$ of that of silica cladding, comparing well with the values published in [31].
Taking into account such a small value and following the same reasoning as for the previous fiber, one can conclude that the temperature sensitivity is negative and increases (absolute value) with increasing temperature. An inversion on this tendency will occur at $\sim 650 \mathrm{~K}$, temperature beyond which the thermal expansion will dominate [10]. The thermal behavior of the PS-LPFG written in the $\mathrm{B} / \mathrm{Ge}$ co-doped fiber is non-linear. In the $5-35 \mathrm{~K}$ temperature range, an average temperature sensitivity of about -0.08 and $0.2 \mathrm{~nm} / \mathrm{K}$ can be considered for the first and the second/third set of experiments, respectively. In general, beyond $60 \mathrm{~K}$ the data points of the two experiments fits well and there are three temperature ranges where a linear fitting can be applied. In the $60-240 \mathrm{~K}$ temperature range, the average temperature sensitivity was of the order of $-0.43 \mathrm{~nm} / \mathrm{K}$. It should be noted the decrease (absolute value) in the temperature sensitivity to about $-0.07 \mathrm{~nm} / \mathrm{K}$ in the range $240-285 \mathrm{~K}$, before increasing again towards an average value of about $-0.38 \mathrm{~nm} / \mathrm{K}$ as one approaches room temperature, a fact apparently not observed in other published works involving LPFGs [11], [32].

\section{CONCLUSION}

We have studied the thermal behavior of PS-LPFG, working in reflection, from $5 \mathrm{~K}$ up to room temperature. The results show that this configuration can be used to implement a compact cryogenic temperature sensor requiring access only to one end of the fiber, as is the case of FBGs, but exhibiting the higher temperature sensitivities of LPFGs for this temperature range. The sensitivities were higher for the PS-LPFG inscribed in the $\mathrm{B} / \mathrm{Ge}$ co-doped fiber when compared to the one written in the SMF28 fiber. In order to clarify the behavior, in particular, of the PS-LPFG inscribed in the B/Ge co-doped fiber at lower temperatures, several improvements are now being implemented, such as the deposition of a metal-coated mirror on the fiber end-face (to increase the optical power reflected and the contrast between Peak and Dips) and the use of an unpolarized light source. A set of heating cycles for the whole temperature range will be performed to check for sensor repeatability. In addition, a study of the wavelength stability against time will be conducted in order to find the source of the sensor inaccuracy. The temperature sensitivity can be further increased at temperatures below $60 \mathrm{~K}$ by using PS-LPFGs in the vicinity of the turning points. As an alternative to PS-LPFGs, reflective bandpass LPFGs are also been studied [33]. It should be noted that although the temperature sensitivity can be increased and the sensor reliability problem overcome, due to the relatively large bandwidth of the resonances, to attain the temperature resolution published in literature for other systems will be a considerable challenge [34]. A final remark to the time response of the sensor that can be improved in a practical situation by using a silica capillary (to prevent direct contact with the cryogenic fluid) which has higher thermal conductivity.

\section{ACKNOWLEDGMENT}

The authors acknowledge operation NORTE-070124 FEDER-000070-MULTIFUNCTIONAL NANOMATERIALS for financial support. J.H. Belo thanks FCT for the Phd grant. 


\section{REFERENCES}

[1] T. Chen, D. Xu, M. Buric, M. Maklad, P. R. Swinehart, and K. P. Chen, "Self-heated all-fiber sensing system for cryogenic environments," Meas. Sci. Technol., vol. 21, no. 9, pp. 094036-1-094036-8, Jul. 2010.

[2] T. Habisreuther, E. Hailemichael, W. Ecke, I. Latka, K. Schröder, C. Chojetzki, K. Schuster, M. Rothhardt, and R. Willsch, "ORMOCER coated fiber-optic Bragg grating sensors at cryogenic temperatures," IEEE Sensors J., vol. 12, no. 1, pp. 13-16, Jan. 2012.

[3] (2014, 17 jan.). [Online]. Available: www.iter.org

[4] R. Rajinikumar, M. Süßer, K. G. Narayankhedkar, G. Krieg, and M. D. Atrey, "Design parameter evaluation of a metal recoated Fiber Bragg Grating sensors for measurement of cryogenic temperature or stress in superconducting devices," Cryogenics, vol. 49, no. 5, pp. 202-209, May 2009.

[5] Z.-S. Guo, J. Feng, and H. Wang, "Cryogenic temperature characteristics of the fiber Bragg grating sensors," Cryogenics, vol. 52, no. 10, pp. 457-460, Oct. 2012.

[6] Y. Tanaka, M. Ogata, K. Nagashima, H. Agawa, S. Matsuura, and Y. Kumagai, "Experimental investigation of optical fiber temperature sensors at cryogenic temperature and in high magnetic fields," Phys. C, vol. 470, no. 20, pp. 1890-1894, Nov. 2010.

[7] J. M. López-Higuera, L. R. Cobo, A. Q. Incera, and A. Cobo, "Fiber optic sensors in structural health monitoring," J. Lightw. Technol., vol. 29, no. 4, pp. 587-608, Feb. 2011.

[8] R. A. Freitas, "Optical fiber temperature sensors for cryogenic applications," M.S. thesis, Faculdade de Ciências da Universidade do Porto, Departamento de Física, Porto, Portugal, 2012.

[9] V. Bathia, "Applications of long-period gratings to single and multiparameter sensing," Opt. Exp., vol. 4, no. 11, pp. 457-466, May 1999.

[10] G. M. Rego, "Arc-induced long-period fibre gratings: "Fabrication and their applications in optical communications and sensing," $\mathrm{Ph}$.D. dissertation, Faculdade de Engenharia da Universidade do Porto, Departamento de Engenharia Eletrotécnica e de Computadores, Porto, Portugal, 2006.

[11] S. W. James, R. P. Tatam, A. Twin, R. Bateman, and P. Noonan, "Cryogenic temperature response of fibre optic long period gratings," Meas. Sci. Technol., vol. 14, no. 8, pp. 1409-1411, Aug. 2003.

[12] J. Monteiro, R. Martins, P. Caldas, and G. Rego, "Fiber optic sensor based on long period fiber grating to monitor cryogenic temperatures," in Proc. IX SEONS, Jun. 2011, Post Deadline Paper.

[13] J. Huang, X. Lan, A. Kaur, H. Wang, L. Yuan, and H. Xiao, "Reflectionbased phase-shifted long period fiber grating for simultaneous measurement of temperature and refractive index," Opt. Eng., vol. 52, no. 1, pp. 014404-1-014404-4, Jan. 2013.

[14] R. Martins, J. Monteiro, P. Caldas, J. L. Santos, and G. Rego, "Reflectionbased phase-shifted long-period fiber grating for cryogenic temperature measurements," in Proc. SPIE, 23rd Int. Conf. Opt. Fiber Sensors, Spain, Santander, vol. 9157, June 2-6, 2014, pp. 91571K-1-91571K-4.

[15] H. Ke, K. S. Chiang, and J. H. Peng, "Analysis of phase-shifted longperiod fiber grating," IEEE Photon. Technol. Lett., vol. 10, no. 11, pp. 1596-1598, Nov. 1998.

[16] Y. Liu, J. A. R. Williams, L. Zhang, and I. Bennion, "Phase shifted and cascaded long-period fiber gratings," Opt. Commun., vol. 164, nos. 1-3, pp. 27-31, Jun. 1999.

[17] G. Humbert and A. Malki, "High performance bandpass filters based on electric arc-induced $\pi$-shifted long-period fibre gratings," Electron. Lett., vol. 39 , no. 21 , pp. 1506-1507, Oct. 2003.

[18] L. R. Chen, "Phase-shifted long-period gratings by refractive indexshifting," Opt. Commun., vol. 200, nos. 1-6, pp. 187-191, Dec. 2001.
[19] J. Bae, J. K. Bae, S. H. Kim, S. B. Lee, and J. Chun, "Analysis for longperiod fiber gratings using thermal kernel function," Opt. Exp., vol. 12, no. 5, pp. 797-810, Mar. 2004.

[20] X. Shu, L. Zhang, and I. Bennion, "Sensitivity characteristics of longperiod fiber gratings," J. Lightw. Technol., vol. 20, no. 2, pp. 255-266, Feb. 2002.

[21] S. W. James and R. P. Tatam, "Optical fibre long period grating sensors: Characteristics and application," Meas. Sci. Technol., vol. 14, no. 3, pp. R49-R61, Mar. 2003.

[22] R. Falate, O. Frazão, G. Rego, O. Ivanov, H. J. Kalinowski, J. L. Fabris, and J. L. Santos, "Bending sensitivity dependent on the phase-shift imprinted in the long-period fibre gratings," Meas. Sci. Technol., vol. 18, no. 10, pp. 3123-3130, Oct. 2007.

[23] G. Rego, O. Ivanov, and P. V. S. Marques, "Demonstration of coupling to symmetric and antisymmetric cladding modes in arc-induced long-period fiber gratings," Opt. Exp., vol. 14, no. 21, pp. 9594-9599, Oct. 2006.

[24] D. R. Uhlmann and N. J. Kreidl, Optical Properties of Glasses. Westerville, OH, USA: American Ceramic Society Inc., 1991, p. 19.

[25] R. Rajinikumar, M. Süßer, K. G. Narayankhedkar, G. Krieg, and M. D. Atrey, "Design parameter evaluation of a metal recoated fiber Bragg grating sensors for measurement of cryogenic temperature or stress in superconducting devices," Cryogenics, vol. 49, no. 5, pp. 202-209, May 2009.

[26] H. Yamada, Y. Tanaka, M. Ogata, K. Mizuno, K. Nagashima, S. Okumura, and Y. Terada, "Measurement and improvement of characteristics using optical fiber temperature sensors at cryogenic temperatures," Phys. C, vol. 471, nos. 21/22, pp. 1570-1575, Nov. 2011.

[27] H.-J. Yoon, D. M. Costantini, H. G. Limberger, R. P. Salathé, C.-G. Kim, and V. Michaud, "In situ strain and temperature monitoring of adaptive composite materials," J. Intell. Mater. Syst. Struct., vol. 17, no. 12, pp. 1059-1067, Dec. 2006.

[28] S. Pal, T. Sun, K. T. V. Grattan, S. A. Wade, S. F. Collins, G. W. Baxter, B. Dussardier, and G. Monnom, "Non-linear temperature dependence of Bragg gratings written in different fibres, optimised for sensor applications over a wide range of temperatures," Sens. Actuators A, vol. 112, nos. 2/3, pp. 211-219, May 2004.

[29] G. Ghosh, "Model for thermo-optic coefficients of some standard optical glasses," J. Non-Cryst. Solids, vol. 189, nos. 1/2, pp. 191-196, Aug. 1995.

[30] T. BAAK, "Thermal coefficient of refractive index of optical glasses," J. Opt. Soc. Amer., vol. 59, no. 7, pp. 851-857, Jul. 1969.

[31] J. Ballato and P. Dragic, "Materials developments for next generation of optical fiber," Materials, vol. 7, no. 9R, pp. 4411-4430, Jun. 2014.

[32] D. Choi, J. Kim, S. Roh, B. Lee, and Y. W. Lee, "Highly-sensitive and -linear cryogenic temperature response of long-period fiber gratings written on B-Ge-codoped photosensitive fiber," Jpn. J. Appl. Phys., vol. 51, no. 9R, p. 092501, Aug. 2012.

[33] M. Jiang, A. P. Zhang, Y.-C. Wang, H.-Y. Tam, and S. He, "Fabrication of a compact reflective long-period grating sensor with a cladding-mode-selective fiber end-face mirror," Opt. Exp., vol. 17, no. 20, pp. 17976-17982, Sep. 2009.

[34] X. Lu, M. Soto, and L. Thévenaz, "MilliKelvin resolution in cryogenic temperature distributed fibre sensing based on coherent Rayleigh scattering," in Proc. SPIE, 23rd Int. Conf. Opt. Fiber Sensors, Spain, Santander, June 2-6, 2014, vol. 9157, pp. 91573R-1-91573R-4.

Authors' biographies not available at the time of publication. 